\title{
Hypoxia inducible factor-1 $\alpha$-dependent epithelial to mesenchymal transition under hypoxic conditions in prostate cancer cells
}

\author{
MINGCHUAN LI $^{1 *}$, YONG XING WANG $^{1 *}$, YONG LUO $^{1}$, JIAHUI ZHAO $^{1}$, \\ QING LI $^{1}$, JIAO ZHANG ${ }^{2}$ and YONGGUANG JIANG ${ }^{1}$ \\ ${ }^{1}$ Department of Urology, Beijing Anzhen Hospital, Capital Medical University, Beijing 100029, P.R. China; \\ ${ }^{2}$ Department of Anatomy and Cell Biology, East Carolina University, Greenville, NC 27834, USA
}

Received January 6, 2016; Accepted March 17, 2016

DOI: $10.3892 / o r .2016 .4766$

\begin{abstract}
Prostate cancer is the most commonly diagnosed cancer in men and the second leading cause of cancer death. Hypoxia is an environmental stimulus that plays an important role in the development and cancer progression especially for solid tumors. The key regulator under hypoxic conditions is stabilized hypoxia-inducible factor (HIF)-1 $\alpha$. In the present study, immune-fluorescent staining, siRNAs, qRT-PC, immunoblotting, cell migration and invasion assays were carried out to test typical epithelial to mesenchymal transition under hypoxia and the key regulators of this process in PC3, a human prostate cancer cell line. Our data demonstrated that hypoxia induces diverse molecular, phenotypic and functional changes in prostate cancer cells that are consistent with EMT. We also showed that a cell signal factor such as HIF-1 $\alpha$, which might be stabilized under hypoxic environment, is involved in EMT and cancer cell invasive potency. The induced hypoxia could be blocked by HIF-1 $\alpha$ gene silencing and reoxygenation of EMT in prostate cancer cells, hypoxia partially reversed accompanied by a process of mesenchymal-epithelial reverting transition (MErT). EMT might be induced by activation of HIF-1 $\alpha$-dependent cell signaling in hypoxic prostate cancer cells.
\end{abstract}

\section{Introduction}

Prostate cancer is the most commonly diagnosed cancer in men and the second leading cause of cancer death during the

Correspondence to: Professor Yong-Guang Jiang, Department of Urology, Beijing Anzhen Hospital, Capital Medical University, Anzhenli Street, Chaoyang, Beijing 100029, P.R. China

E-mail: jiangyganzhen@sina.cn

Professor Jiao Zhang, Department of Anatomy and Cell Biology, East Carolina University, Greenville, NC 27834, USA

E-mail: zhangj15@ecu.edu

*Contributed equally

Key words: prostate cancer, hypoxia, HIF-1 $\alpha$, mesenchymalepithelial transition, mesenchymal-epithelial reverting transition past 5 years according to the cancer statistics (1) reported by the American Cancer Society, this trend can be traced back even 10 years. For patients with localized prostate cancer, it is treatable and the 5-year survival rates could be even $100 \%$. Yet, patients usually die of cancer metastasis or drug resistance with the 5-year survival rates lowered to $30-40 \%$. It is urgent to explore the mechanisms involved in cancer metastasis and drug resistance and develop new strategies to improve the treatment outcome.

Hypoxia is an environmental stimulus that plays an important role in cancer development and progression especially in solid tumors which outgrow local blood supply during the progression to advanced stages. Hypoxic conditions are widely present in many human malignancies including breast, prostate, lung, pancreas, rectum and renal cell cancer (2). The key regulator under hypoxic conditions is stabilized hypoxiainducible factor (HIF)-1 $\alpha$ which dimerizes with constitutively expressed HIF-1 $\beta$ and then translocates into the nucleus where they bind to a specific sequence, the hypoxia-responsive element (HRE), usually present in the promoter of several hypoxia-dependent target genes (3).

Epithelial to mesenchymal transition (EMT) was first used to depict embryonic development, which is characterized by adherent epithelial cells converting to motile mesenchymal cells. EMT is now been classified into 3 different subtypes that is EMT during implantation, embryogenesis and organ development, EMT associated with tissue regeneration and organ fibrosis, EMT associated with cancer progression and metastasis (4). It has been reported that EMT existed in many kinds of human cancers such as pancreatic, breast and colon cancer (5) and hypoxia alone can trigger an EMT process with increased invasiveness (5). At the molecular level, EMT is accompanied by loss of epithelial cell markers, such as cell adhesion protein E-cadherin, and acquisition of mesenchymal markers, such as vimentin and $\mathrm{N}$-cadherin. With more and more research concerning on the role of EMT in cancer progression and metastasis, a family of transcriptional factors including Snail, Slug, Twist, Zeb and E47 have emerged as EMT master genes since they can directly downregulate E-cadherin expression which is a hallmark of EMT.

As mentioned above, hypoxia alone can trigger EMT in many solid tumors including hepatoblastoma, pancreatic, colon and breast cancer (5), yet, the mechanisms involved in 
prostate cancer under hypoxic condition remain unclear. In the present study, we demonstrated that hypoxia might induce diverse molecular, phenotypic, and functional changes in prostate cancer cells that are consistent with EMT. We also showed that cell signaling factors such as HIF-1 $\alpha$, which is thought to be stabilized under hypoxic environment is involved in EMT and cancer cell invasive potency. Hypoxia-induced EMT in prostate cancer cells can be blocked by HIF-1 $\alpha$ gene silencing and reoxygenation after hypoxia partially reverses EMT which is accompanied by a process named mesenchymal-epithelial reverting transition (MErT). We conclude that EMT could be induced by a mechanism that might involve the activation of HIF-1 $\alpha$-dependent cell signaling in hypoxic prostate cancer cells.

\section{Materials and methods}

Cell culture under normal and hypoxic conditions. Human prostate cancer cell lines PC3 and DU145 were purchased from the National Platform of Experimental Cell Resources for Sci-Tech (Beijing, China). The cells were cultured in RPMI1640 medium supplemented with $10 \%$ fetal bovine serum (FBS; Gibco-BRL, Grand Island, NY, USA) at $37^{\circ} \mathrm{C}$ under a $5 \% \mathrm{CO}_{2}$ condition. To recapitulate the effects of hypoxia as it occurs in prostate cancer, we exposed $60-70 \%$ subconfluent PC3 cells to hypoxic conditions $\left(3 \% \mathrm{O}_{2}, 5 \% \mathrm{CO}_{2}\right.$ and $\left.92 \% \mathrm{~N}_{2}\right)$ for up to $72 \mathrm{~h}$.

Fluorescent immunostaining. Immunofluorescence staining was employed to further confirm EMT phenotype changes of prostate cancer cells under hypoxic condition. PC3 and DU145 cells were cultured on coverslips in 24-well plates at initial density $5 \times 10^{4}$ cells/well. At the indicated time-points, culture media were removed from the cultured cells followed by 3 washings with PBS. Cells were fixed with $4 \%$ polyoxymethylene solution for $20 \mathrm{~min}$ and washed with PBS 3 times. Cells were incubated with rabbit anti-human E-cadherin polyclonal antibody (Santa Cruz Biotechnology, Santa Cruz, CA, USA), and mouse anti-human vimentin monoclonal antibody (Santa Cruz Biotechnology) respectively, then their corresponding lumophore conjugated secondary antibodies. DAPI was used for nuclei staining. Finally, cells were observed under a fluorescent microscope or a confocal microscope.

Plasmid construction and transfections. PcDNA3.1 plasmid (a generous gift from Professor Dalin He) was digested with endonucleases HindIII and BglII in order to construct vectors for expression of HIF- $1 \alpha$-small interfering RNAs (siRNAs). Three chemically synthesized oligonucleotides encoding HIF-1 $\alpha$-short hairpin siRNAs that included a loop motif were inserted downstream of the BglII promoter of the plasmid using DNA Ligation kit (Takara Biotechnology, Co., Ltd., Dalian, China) and cloned. PC3 cells were transfected with the above plasmids (4 $\mu \mathrm{g}$ total) using Lipofectamine ${ }^{\mathrm{TM}} 2000$ (Invitrogen Corp., Carlsbad, CA, USA) according to the manufacturer's instructions. A scramble siRNA was used as control.

Quantitative real-time PCR ( $q R T-P C R)$. PC3 and DU145 cells were harvested at the indicated time-points. Total RNA was extracted by using TRIzol (Invitrogen) according to the
Table I. Primers used for the analysis.

Primers

\begin{tabular}{|c|c|}
\hline Snail & $\begin{array}{l}\text { Forward: CCACGAGGTGTGACTAACTATG } \\
\text { Reverse: ACCAAACAGGAGGCTGAAATA }\end{array}$ \\
\hline Slug & $\begin{array}{l}\text { Forward: AACTACAGCGAACTGGACAC } \\
\text { Reverse: GAGGATCTCTGGTTGTGGTATG }\end{array}$ \\
\hline Twist & $\begin{array}{l}\text { Forward: AGGCATCACTATGGACTTTCTC } \\
\text { Reverse: GGCCAGTTTGATCCCAGTAT }\end{array}$ \\
\hline Zeb1 & $\begin{array}{l}\text { Forward: CTTCTCACACTCTGGGTCTTATTC } \\
\text { Reverse: CGTTCTTCCGCTTCTCTCTTAC }\end{array}$ \\
\hline Zeb2 & $\begin{array}{l}\text { Forward: CTAACCCAAGGAGCAGGTAATC } \\
\text { Reverse: GTGAATTCGCAGGTGTTCTTTC }\end{array}$ \\
\hline $\mathrm{E} 47$ & $\begin{array}{l}\text { Forward: GTCTCGGTCATCCTGAACTTG } \\
\text { Reverse: TTTCCTCTTCTCGCCGTTTC }\end{array}$ \\
\hline GAPDH & $\begin{array}{l}\text { Forward: GGTGTGAACCATGAGAAGTATGA } \\
\text { Reverse: GAGTCCTTCCACGATACCAAAG }\end{array}$ \\
\hline
\end{tabular}

manufacturer's protocol. Reverse transcription was performed according to the protocol of SuperScript ${ }^{\mathrm{TM}}$ First-Strand Synthesis System for RT-PCR (Invitrogen). Quantitative real-time PCR was performed using SYBR Premix Ex Taq (Takara Biotechnology) under conditions recommend by the manufacturer and Applied Biosystems 7300 Real-Time PCR System (Applied Biosystems, Waltham, MA, USA) supplied with the analytical software.

Primers were designed for transcription factors that regulate EMT, including Snail, Slug, Twist, Zeb1, Zeb2 and E47, GAPDH gene as an internal reference gene, by using web-based program at www.idtdna.com and synthesized from Integrated DNA Technologies (Coralville, IA, USA). The primers used for the analysis are shown in Table I.

Immunoblotting with ECL detection. PC3 cells were incubated under hypoxic conditions for different periods of time $(6,24$, 48 and 72 h). After each indicated incubation period, PC3 cells for protein assay were lysed in RIPA buffer (1\% Triton X-100, $0.5 \%$ deoxycholic acid, $0.2 \%$ SDS, $150 \mathrm{mM}$ sodium chloride and $2 \mathrm{mM}$ EDTA) with complete protease inhibitor cocktail tablet (Roche, Mannheim, Germany) and pepstatin A. After removing cell debris by centrifugation, protein concentration was determined using BCA method.

Total proteins were separated on $8-12 \%$ polyacrylamide gels and transferred onto $0.45 \mu \mathrm{m}$ nitrocellulose in a buffer containing 25 mmol/1 Tris- $\mathrm{HCl}$ (pH 8.3), $192 \mathrm{mmol} / \mathrm{l}$ glycine, $20 \%$ methanol and blocked with 5\% fat-free dry milk in PBS for $2 \mathrm{~h}$. The membranes were incubated with primary antibodies. The following antibodies were used: rabbit anti-human HIF-1 $\alpha$ polyclonal antibody (Santa Cruz Biotechnology), rabbit anti-human E-cadherin polyclonal antibody (Santa Cruz Biotechnology), and mouse anti-human vimentin monoclonal antibody (Santa Cruz Biotechnology). $\beta$-actin was used as internal control. 
A

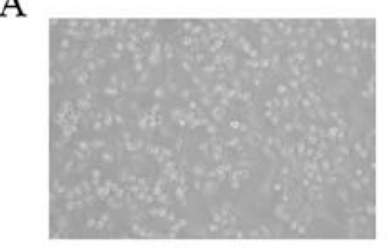

Normal

B

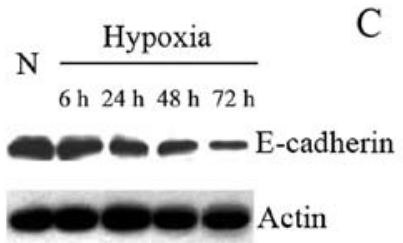

$\mathrm{D}$
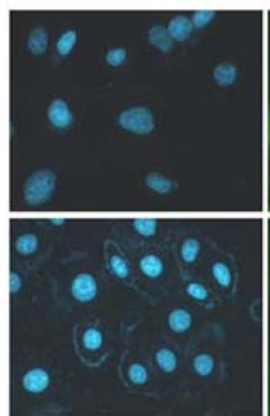

DAPI

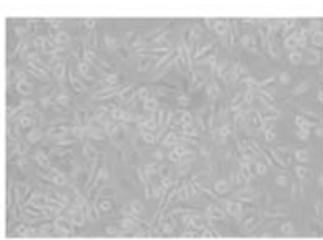

Hypoxia

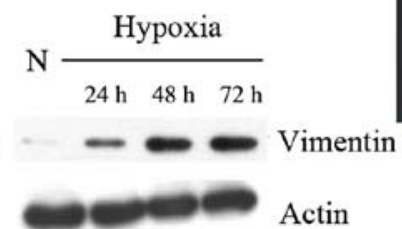

$\mathrm{F}$

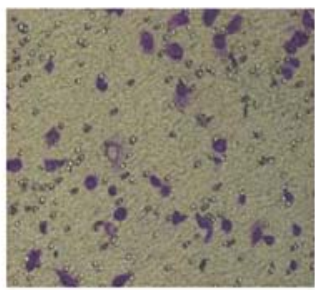

Normoxia (12 h)

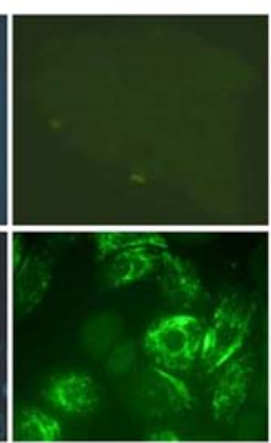

Vimentin
Normoxia

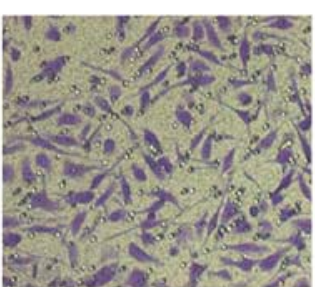

Hypoxia (12 h)
Normoxia

Hypoxia

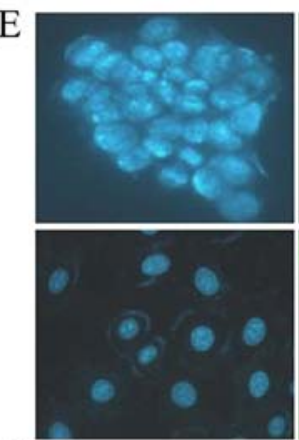

DAPI
Hypoxia

$\sqrt{2}+x^{2}$

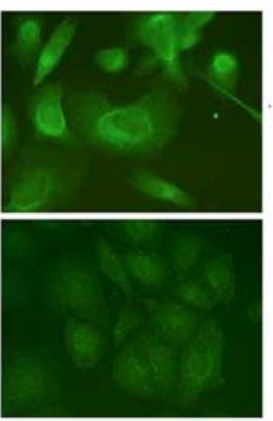

E-cadherin

Figure 1. Hypoxia induces EMT phenotype in prostate cancer cells. (A) Cells were cultured for $72 \mathrm{~h}$ in $21 \% \mathrm{O}_{2}$ (normoxia; $\mathrm{N}$ ) or $3.0 \% \mathrm{O}_{2}$ (hypoxia; $\mathrm{H}$ ). Cell images were captured by phase-contrast microscopy. (B) Western blot analysis of epithelial marker (E-cadherin) under normoxic (N) or hypoxic conditions. A representative blot from 3 independent experiments is shown. (C) Western blot analysis of mesenchymal marker (vimentin) under normoxic (N) or hypoxic conditions. A representative blot from 3 independent experiments is shown. (D) Immunofluorescence staining of E-cadherin in PC 3 under normoxic or hypoxic conditions for $72 \mathrm{~h}$. Green represents E-cadherin staining. Blue signal represents nuclear DNA staining by DAPI (original magnification, $\mathrm{x} 400$ ). (E) Immunofluorescence staining of vimentin in PC3 under normoxic or hypoxic conditions for $72 \mathrm{~h}$. Green signal represents vimentin staining. Blue signal represents nuclear DNA staining by DAPI (original magnification, $\mathrm{x} 400$ ). (F) Matrigel invasion assay. Photomicrographs showing cells that passed through Matrigel under normoxic or hypoxic conditions for $12 \mathrm{~h}$ (original magnification, x100).

Cell migration and invasion assays. The invasion assays were performed using Millicell inserts (Millipore, Billerica, MA, USA) coated with Matrigel (BD Biosciences, Sparks, MD, USA). Cells $\left(2.5 \times 10^{4}\right)$ were seeded per upper chambers in serum-free DMEM whereas the lower chambers were loaded with DMEM containing 5\% FBS. After $12 \mathrm{~h}$, on the upper chambers non-migrating cells were removed by cotton swabs, and cells invaded through the Matrigel layer to the underside of the membrane were stained by crystal violet. The cell numbers were counted. Cell migration assays were performed similarly, but without Matrigel.

Statistical analysis. Each experiment was performed at least 3 times. All values are presented as mean \pm SD. The statistics were analyzed by unpaired, two-tailed t-test. Data were considered to be statistically significant when $\mathrm{P}<0.05$.

\section{Results}

Hypoxia results in the morphologic and cell biological changes characteristic of EMT in prostate cancer cells. After $72 \mathrm{~h}$ culture at hypoxic conditions $\left(3 \% \mathrm{O}_{2}, 5 \% \mathrm{CO}_{2}\right.$ and $92 \%$ N2), PC3 cells started to loose cell contacts, scattered from cell clusters and acquired a spindle-shaped and fibroblast-like

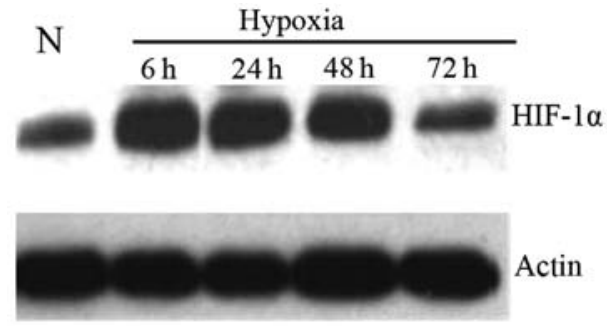

Figure 2. Hypoxia induced HIF-1 $\alpha$ overexpression in prostate cancer cells Western blot analysis of epithelial marker (E-cadherin) under normoxic (N) or hypoxic conditions. A representative blot from 3 independent experiments is shown.

phenotype which is characteristic of EMT compared to the normoxic counterparts (95\% air and $5 \% \mathrm{CO}_{2}$ ) (Fig. 1A).

To further confirm whether prostate cancer underwent EMT, the expression of markers of epithelial and mesenchymal phenotypes were detected by western blot analysis (Fig. 1B and C). As is shown in Fig. 1B and C, hypoxic PC3 cells underwent a typical transition manifested by reduced E-cadherin expression and increased vimentin expression compared with normoxic control.

Immunofluorescence staining showed that the expression of E-cadherin decreased (Fig. 1D), but the expression of vimentin 


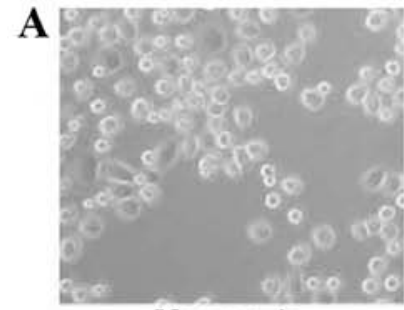

Normoxia

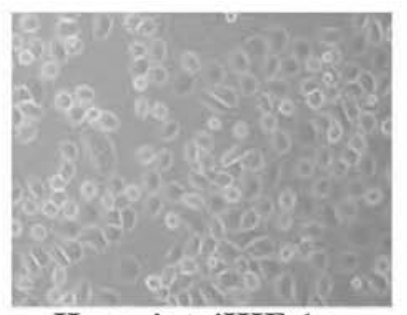

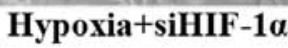

\section{N $\frac{\text { HYP+siHIF-1a }}{24 \text { h } 48 \text { h } 72 \text { h }}$}

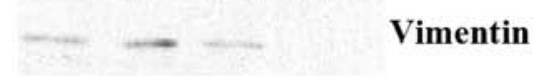

Actin

D

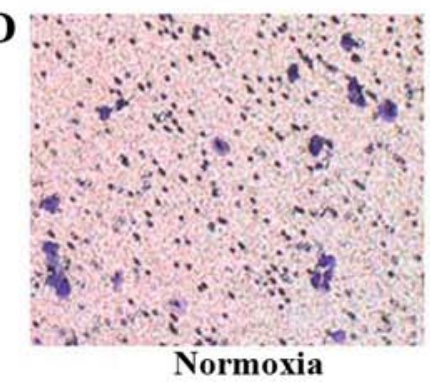

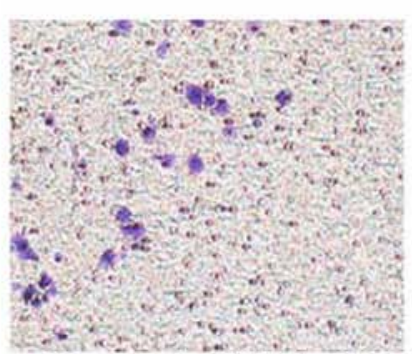

Hypoxia+siHIF-1 $\alpha(12$ h)

Figure 3. Inhibition of HIF- $1 \alpha$ reverses EMT characteristics in prostate cancer cells under hypoxic conditions. (A) Cells were cultured for 48 h in $21 \%$ O (normoxia) or $3.0 \% \mathrm{O}_{2}$ (hypoxia) with additional siHIF-1 $\alpha$ treatment. Cell images were captured by phase-contrast microscopy. (B) Western blot analysis. No obvious change was observed in E-cadherin protein expression when treated with siHIF-1 $\alpha$ under hypoxia compared to culture under normoxia. (C) Western blot analysis. No obvious change in vimentin protein expression when treated with siHIF-1 $\alpha$ under hypoxia compared to culture under normoxia. (D) Matrigel invasion assays. Cells that have passed through Matrigel under normoxic or hypoxic conditions with siHIF-1 $\alpha$ treatment for $12 \mathrm{~h}$ (original magnification, $\mathrm{x} 100$ ) showed no significant differences.
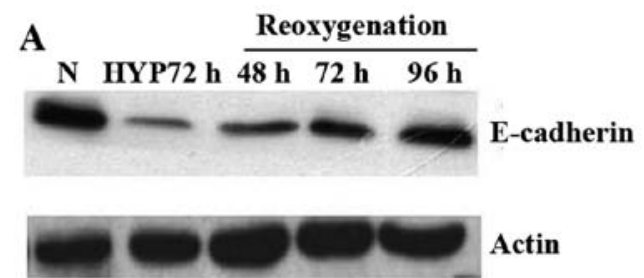

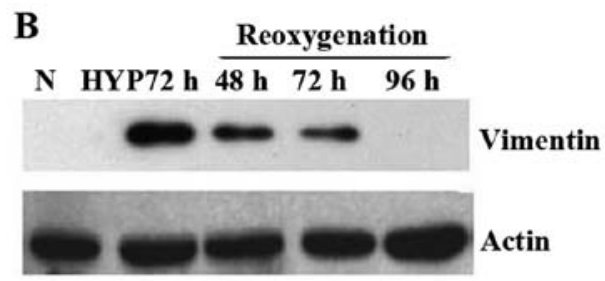

Figure 4. Reoxygenation reverses EMT characteristics in prostate cancer cells. (A) Western blot analysis. Increased E-cadherin protein expression when re-cultured under normoxia. (B) Western blot analysis. Decreased vimentin protein expression when re-cultured under normoxia.

increased (Fig. 1E) when compared with their counterparts in normoxic environment. Matrigel invasion assay was also used to assess the cell invasiveness, which indicated that PC3 cells under hypoxic conditions for $12 \mathrm{~h}$ readily migrated through the Matrigel chamber in a relatively high numbers, whereas their normoxic partners exhibited a less invasive potency (Fig. 1F).

Under hypoxic conditions prostate cancer cells exhibit heightened HIF-1 $\alpha$ expression. HIF-1 $\alpha$ was analyzed by western blot analysis to determine whether the EMT observed in prostate cancer cells was attributable to heightened HIF-1 $\alpha$ activity under hypoxic conditions (Fig. 2). PC3 cells were incubated under hypoxic conditions and collected as described before. As shown by western blot analysis (Fig. 2), HIF-1 $\alpha$ protein levels in prostate cancer cells were upregulated during hypoxia and reached the highest expression at $6 \mathrm{~h}$. The increased HIF-1 $\alpha$ protein level was possibly due to the variation of protein stability under hypoxia condition.

Inhibition of HIF-1 $\alpha$ activity in prostate cancer cells under hypoxic conditions or reoxygenation reverses EMT. Having established that hypoxia results in elevated HIF-1 $\alpha$ expression

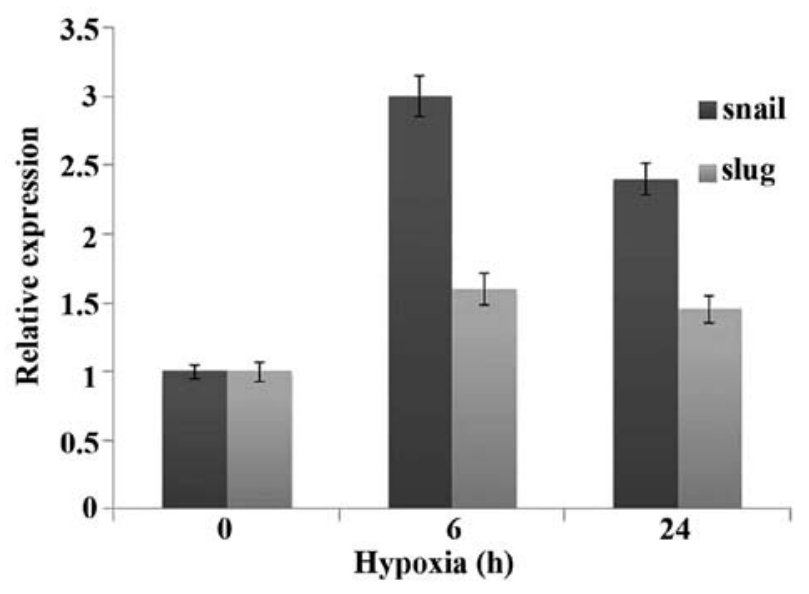

Figure 5. Relative expression of Snail and Slug under hypoxia in PC3 cells. Prostate cancer cells under hypoxic conditions exhibit upregulation of Snail and Slug, transcriptional regulators of the EMT program.

in prostate cancer cells, we next investigated the possibility for inhibition of HIF-1 $\alpha$ to attenuate the mesenchymal characteristics of hypoxic cells. Molecular inhibition was 


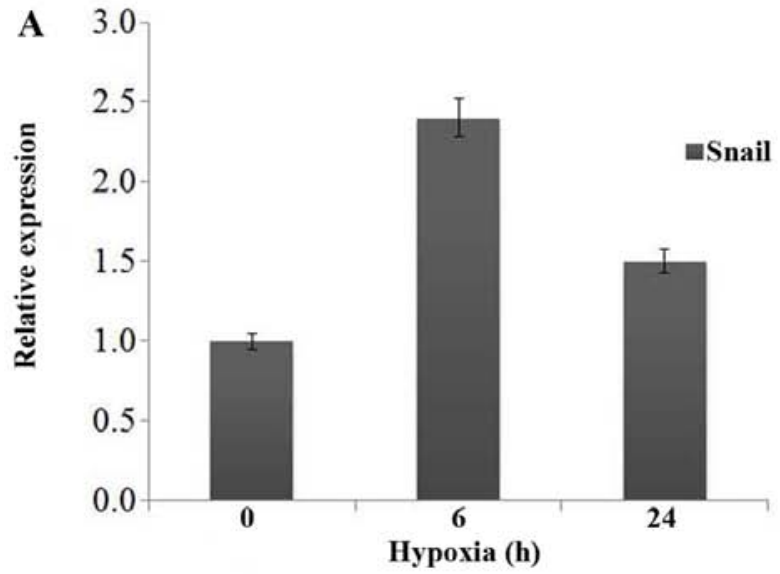

B

Figure 6. Hypoxic conditions upregulate the invasive capability of epithelial-origin mesenchymal cells. (A) Relative expression of snail under hypoxia in DU145 cells. (B) Transwell assay under hypoxia in DU145 cells.

accomplished by siRNA to downregulate HIF-1 $\alpha$. Inhibition of HIF- $1 \alpha$ activity by siRNA under hypoxic conditions for $48 \mathrm{~h}$ did not cause any significant changes in cell morphology (Fig. 3A). No obvious change in protein expression was detected by western blot analysis (Fig. 3B and C), consistent with reversion to an epithelial phenotype. Importantly, inhibition of HIF-1 $\alpha$ by siRNA led to a remarkable decrease in the invasiveness of hypoxic cells compared with control in Matrigel invasion assay at $12 \mathrm{~h}$ under hypoxic conditions (Fig. 3D).

At the same time, we elucidated what would happen when PC3 cells were re-cultured under normoxic conditions after exposed to hypoxic conditions for $72 \mathrm{~h}$. Western blot analysis showed that E-cadherin protein was regained when PC3 cells returned to normoxia gradually compared to those cultured under hypoxia (Fig. 4A), whereas expressions of vimentin gradually lost in contrast to the enhanced expression under hypoxia condition (Fig. 4B). These changes occurred after inhibition of HIF-1 $\alpha$ activity in PC3 cells indicated that prostate cancer cells might undergo MErT through reoxygenation after they were exposed in hypoxic conditions.

Prostate cancer cells under hypoxic conditions exhibit upregulation of Snail and Slug, transcriptional regulators of the EMT program. The gene expression cataract that mediates EMT is regulated by one or more transcription factors, including Snail, Slug, Twist, Zeb1, Zeb2 and E47 (6-9) and these transcription factors are transcriptionally induced by upstream signals, including hypoxia or HIF-1 $\alpha$. Real-time PCR result showed that, hypoxic prostate cancer cells exhibited substantial upregulation of Snail and Slug compared with normoxic cells (Fig. 5).

Hypoxic conditions upregulated the invasive capability of epithelial-origin mesenchymal cells. The DU145 cell line was derived from prostate cancer brain metastasis, which showed fibroblastic-like phenotype under normoxia condition. The spindle shape of DU145 cells did not show any change when they were cultured under hypoxic environment, but real-time PCR results showed that DU145 cells exhibited substantial upregulation of Snail as compared with normoxic cells (Fig. 6A). Transwell assay also showed that DU145 has remarkably increased invasiveness, which related to the activation of HIF-1 $\alpha$ signal pathway and the upregulation of Snail (Fig. 6B) under hypoxic conditions.

\section{Discussion}

EMT has been defined as a three-part process in which cells firstly acquire a fibroblast-like morphology, and then downregulation of epithelial-specific proteins such as E-cadherin while simultaneously expression of mesenchymal proteins such as vimentin, and ultimately digest and migrate through extracellular matrix (ECM) (10). Hypoxia alone can trigger EMT in many solid tumors including hepatoblastoma, pancreatic, colon and breast cancer (5). Yet, the mechanisms involved in prostate cancer under hypoxic conditions remained unclear. A variety of physiological or pathophysiological conditions can cause imbalance in oxygen supply and demand. In response to reduction in oxygen supply, tissues initiate signaling events that trigger the upregulation of genes that are used to be 'silent' under normoxic conditions to allow for both short and longterm adaptation to hypoxia. Many of these events are initiated by the activation of the hypoxia-inducible factor (HIF) family of transcription factors of which HIF- $1 \alpha$ plays an important role. Thus, we sought to determine whether the EMT observed in prostate cancer cells under hypoxic conditions attribute to enhance HIF-1 $\alpha$ activity.

HIF-1 $\alpha$ was analyzed to determine whether increased HIF-1 $\alpha$ activity result in EMT in prostate cancer cells under hypoxic conditions. PC3 cells were incubated under hypoxic conditions for different periods of time (6, 24, 48 and $72 \mathrm{~h}$ ) and western blot analysis was used to prove our hypothesis. Having established that hypoxia lead to elevated HIF-1 $\alpha$ expression in prostate cancer cells, we next investigated the possibility for inhibition of HIF- $1 \alpha$ to attenuate the mesenchymal characteristics of hypoxic cells. Towards this end, siRNAs were used to inhibit HIF-1 $\alpha$ expression in these hypoxic cells and then compared their phenotype with their counterparts. Our data indicated changes in morphology, protein expression and invasion and supported the notion that hypoxia leads to EMT in prostate cancer cells. 
Substantial evidence illustrated that EMT plays important roles in cancer metastasis and is also responsible for resistance to conventional chemotherapeutics (11). Tumor microenvironments, including hypoxia, have been documented as inducing this phenomenon through upregulation of Twist, Snail, Slug, Zeb1 and Zeb2 according to different cancer cell types $(8,12,13)$. Hypoxic microenvironment can be found in central region of solid tumors and intratumoral hypoxia is conducive to high-grade and high invasive ability tumor cell screen which might contribute to tumor malignant progression (14-16). According to epidemiological and clinical studies, hypoxia and hypoxia-induced signaling pathways were associated with poor prognosis of patient including prostate cancer (17), and HIF-1 $\alpha$ was reported to mediate hypoxia and $\operatorname{EMT}(5,9)$. However, the molecular mechanism of hypoxia that induced aggressiveness of prostate cancer has not been defined previously.

It was demonstrated that transcription factor HIF-1 is a heterodimer that composed of a constitutively expressed HIF-1 $\beta$ subunit and a HIF-1 $\alpha$ subunit that mediates adaptive responses to changes in tissue oxygenation (3). The level of HIF-1 $\alpha$ expression is determined not by the rates of protein synthesis but the protein degradation. In the case of normoxia, HIF- $1 \alpha$ protein is degraded by $\mathrm{O}_{2}$-dependent prolylhydroxylation, which targets the protein for ubiquitylation by E3 ubiquitin-protein ligases. Stabilization of HIF-1 $\alpha$ is critical in the transcriptional response to hypoxia. Our preliminary study suggested that high expression of HIF-1 $\alpha$ can induce prostate cancer LnCap to undergo EMT under normoxia (18) and inhibition of $\beta$-catenin through shRNA causes a reversal of EMT and metastatic phenotypes induced by HIF-1 $\alpha$ (19).

Some relevant research has already demonstrated EMT and HIF-1 $\alpha$ in the different human PCa cell lines, for example, DU145, PC-3, PPC-1 and TSU. In these investigations, the majority of EMT studies in prostate cancer used PC-3 and DU145 human prostate cancer cells. Our previous research also included different human PCa cell lines. At the same time, we already successfully proved that overexpression of hypoxia inducible factor- $1 \alpha$ (HIF-1 $\alpha)$ could induce EMT in LNCaP cells, but not in PC3 (18-24). Based on these studies, in the present study, we simulated the hypoxia condition and explored the potential mechanism of HIF-1 $\alpha$ on EMT in human prostate cancer cell lines PC3 and DU145.

In the present study, we confirmed that hypoxia treatment may induce epithelial origin of the prostate cancer cells PC3 to undergo typical EMT transforming, which includes changes in cell morphology, decreased expression of E-cadherin, increased stromal protein vimentin expression, high expression of Snail, accompanied by increased cell invasion and metastasis. Further research indicated that inhibition of HIF-1 $\alpha$ expression under hypoxic conditions or reoxygenation reverses EMT which implies that HIF-1 $\alpha$ plays a critical role under hypoxia induced EMT of prostate cancer and that the EMT process is a means, not an end for cancer metastasis. Our result is consistent with the study by Yang et al (8) who also confirmed that Twist is directly regulated by HIF-1 $\alpha$ to induce tumor cell EMT transformation, while our result indicated that Snail is highly expressed in the hypoxic microenvironment, and its direct inhibition of E-cadherin expression in ovarian tumors were reported (7). Whether Snail mediated EMT phenomenon induced by activation of HIF-1 $\alpha$ under hypoxia, or hypoxia directly regulates the expression of Snail, or whether there is a direct transcriptional regulation between HIF-1 $\alpha$ and Snail requires further experimental studies. HIF-1 $\alpha$ can also induce epithelial cell EMT transformation through other signaling pathways, such as the direct or indirect regulation of vimentin and fibronectin (25). In addition to HIF-1 $\alpha$, there are some other transcriptional factors that can induce EMT phenomenon under hypoxic conditions, such as regulation of E-cadherin and $\beta$-catenin by URG11 (upregulated gene 11) (26) and regulation of GSK $3 \beta$ through hypoxia (5) which can target Snail (27) and further induce EMT.

Phenomena were reported which described a mesenchymal to epithelial reverting transition (MErT), where mesenchymallike prostate cancer cell lines revert to epithelial-like, and re-establish cellular adhesion during colonization when re-express E-cadherin in the liver tumor microenvironment (28-32). There is a report that hyperoxic treatment induces MErT in a rat adenocarcinoma model (33), so partial pressure of oxygen alone can make the tumor cells of epithelial origin conversion between EMT and MErT and it is of great importance to elucidate the mechanisms involved in oxygen pressure changes.

We may conclude that EMT is essential for tumor metastasis and MErT is conducive to the formation of metastasis. MErT transformation conditions might change when the re-expression of E-cadherin and the increase in the ability of the intercellular adhesion is essential. At the same time, we observed that not all cells underwent conversion of MErT accompanying with the time extension of reoxygenation, there are still part of the cells showing interstitial cells long spindle shape and related proteins were not restored to the level prior to hypoxia, which suggested that possibly only certain cells undergo some changes, such as having characteristics of stem cells (30,34-37), but not the conversion of MErT.

The evidence provides incentives for further investigation and optimization in establishing the mechanistic role of how HIF-1 $\alpha$ and Snail work in the attenuation of EMT characteristics and drug resistance under hypoxia and their utility in the clinical practice in the treatment of prostate cancer for which there is no effective and curative therapy.

In conclusion, in the present study, we conclude that EMT could be induced by a mechanism that might involve the activation of HIF-1 $\alpha$-dependent cell signaling in hypoxic prostate cancer cells. Our results provide incentives for further investigation and optimization in establishing the mechanistic role of how HIF-1 $\alpha$ and Snail work in the attenuation of EMT characteristics and drug resistance under hypoxia and their utility in the clinical practice in the treatment of prostate cancer for which there is no effective and curative therapy.

\section{Acknowledgements}

We thank Yatong Chen and Tao Peng for their technical support in research design, acquisition of data, or analysis and interpretation of data. The present study is supported by funds from the National Natural Science Foundation of China (81341066) and the Beijing Health System Special Foundation for building high-level health personnel. 


\section{References}

1. Siegel R, Naishadham D and Jemal A: Cancer statistics, 2013. CA Cancer J Clin 63: 11-30, 2013.

2. Vaupel P and Mayer A: Hypoxia in cancer: Significance and impact on clinical outcome. Cancer Metastasis Rev 26: 225-239, 2007.

3. Semenza GL: Targeting HIF-1 for cancer therapy. Nat Rev Cancer 3: 721-732, 2003.

4. Kalluri R and Weinberg RA: The basics of epithelial-mesenchymal transition. J Clin Invest 119: 1420-1428, 2009.

5. Cannito S, Novo E, Compagnone A, Valfrè di Bonzo L, Busletta C, Zamara E, Paternostro C, Povero D, Bandino A, Bozzo F, et al: Redox mechanisms switch on hypoxia-dependent epithelial-mesenchymal transition in cancer cells. Carcinogenesis 29: 2267-2278, 2008.

6. Peinado H, Olmeda D and Cano A: Snail, Zeb and bHLH factors in tumour progression: An alliance against the epithelial phenotype? Nat Rev Cancer 7: 415-428, 2007.

7. Imai T, Horiuchi A, Wang C, Oka K, Ohira S, Nikaido T and Konishi I: Hypoxia attenuates the expression of E-cadherin via up-regulation of SNAIL in ovarian carcinoma cells. Am J Pathol 163: 1437-1447, 2003.

8. Yang MH, Wu MZ, Chiou SH, Chen PM, Chang SY, Liu CJ, Teng SC and Wu KJ: Direct regulation of TWIST by HIF-1alpha promotes metastasis. Nat Cell Biol 10: 295-305, 2008.

9. Cheng ZX, Sun B, Wang SJ, Gao Y, Zhang YM, Zhou HX, Jia G, Wang YW, Kong R, Pan SH, et al: Nuclear factor- $\kappa \mathrm{B}-$ dependent epithelial to mesenchymal transition induced by HIF-1 $\alpha$ activation in pancreatic cancer cells under hypoxic conditions. PLoS One 6: e23752, 2011.

10. Grünert $S$, Jechlinger $M$ and Beug $H$ : Diverse cellular and molecular mechanisms contribute to epithelial plasticity and metastasis. Nat Rev Mol Cell Biol 4: 657-665, 2003.

11. Huber MA, Kraut N and Beug H: Molecular requirements for epithelial-mesenchymal transition during tumor progression. Curr Opin Cell Biol 17: 548-558, 2005.

12. Min C,Eddy SF, Sherr DH and Sonenshein GE: NF-kappaB and epithelial to mesenchymal transition of cancer. J Cell Biochem 104: 733-744, 2008

13. Shin SR, Sánchez-Velar N, Sherr DH and Sonenshein GE: 7,12-dimethylbenz(a)anthracene treatment of a c-rel mouse mammary tumor cell line induces epithelial to mesenchymal transition via activation of nuclear factor-kappaB. Cancer Res 66: 2570-2575, 2006.

14. Harris AL: Hypoxia - a key regulatory factor in tumour growth Nat Rev Cancer 2: 38-47, 2002.

15. Semenza GL: Defining the role of hypoxia-inducible factor 1 in cancer biology and therapeutics. Oncogene 29: 625-634, 2010.

16. Le QT, Denko NC and Giaccia AJ: Hypoxic gene expression and metastasis. Cancer Metastasis Rev 23: 293-310, 2004.

17. Movsas B, Chapman JD, Greenberg RE, Hanlon AL, Horwitz EM, Pinover WH, Stobbe C and Hanks GE: Increasing levels of hypoxia in prostate carcinoma correlate significantly with increasing clinical stage and patient age: An Eppendorf $\mathrm{pO}_{2}$ study. Cancer 89: 2018-2024, 2000.

18. Jiang YG, Luo Y, He DL, Li X, Zhang LL, Peng T, Li MC, Lin YH: Role of Wnt/beta-catenin signaling pathway in epithelial-mesenchymal transition of human prostate cancer induced by hypoxia-inducible factor-1alpha. Int J Urol 14: 1034-1039, 2007.

19. Zhao JH, Luo Y, Jiang YG, He DL and Wu CT: Knockdown of $\beta$-Catenin through shRNA cause a reversal of EMT and metastatic phenotypes induced by HIF-1 $\alpha$. Cancer Invest 29: 377-382, 2011.
20. Zhao L, Jiang YG, Ma J, Luo Y and Zhao JH: Characterization of prostate cancer cell lines and their epithelial-mesenchymal transition in subcutaneous tumors. Zhonghua Nan Ke Xue 17: 314-317, 2011 (In Chinese).

21. Hugo H, Ackland ML, Blick T, Lawrence MG, Clements JA, Williams ED and Thompson EW: Epithelial-mesenchymal and mesenchymal-epithelial transitions in carcinoma progression. J Cell Physiol 213: 374-383, 2007.

22. Thomas R and Kim MH: HIF-1 alpha: A key survival factor for serum-deprived prostate cancer cells. Prostate 68: 1405-1415, 2008.

23. Zhong H, Agani F, Baccala AA, Laughner E, RiosecoCamacho N, Isaacs WB, Simons JW and Semenza GL: Increased expression of hypoxia inducible factor-1alpha in rat and human prostate cancer. Cancer Res 58: 5280-5284, 1998.

24. Luo Y, He DL, Ning L, Shen SL, Li L, Li X, Zhau HE and Chung LW: Over-expression of hypoxia-inducible factor-1alpha increases the invasive potency of LNCaP cells in vitro. BJU Int 98: 1315-1319, 2006

25. Krishnamachary B, Berg-Dixon S, Kelly B, Agani F, Feldser D, Ferreira G, Iyer N, LaRusch J, Pak B, Taghavi P, et al: Regulation of colon carcinoma cell invasion by hypoxia-inducible factor 1 . Cancer Res 63: 1138-1143, 2003.

26. Du R, Huang C, Bi Q, Zhai Y, Xia L, Liu J, Sun S and Fan D: URG11 mediates hypoxia-induced epithelial-to-mesenchymal transition by modulation of E-cadherin and beta-catenin. Biochem Biophys Res Commun 391: 135-141, 2009.

27. Zhou BP, Deng J, Xia W, Xu J, Li YM, Gunduz M and Hung MC Dual regulation of Snail by GSK-3beta-mediated phosphorylation in control of epithelial-mesenchymal transition. Nat Cell Biol 6: 931-940, 2004

28. Yates CC, Shepard CR, Stolz DB and Wells A: Co-culturing human prostate carcinoma cells with hepatocytes leads to increased expression of E-cadherin. Br J Cancer 96: 1246-1252, 2007.

29. Yates C, Shepard CR, Papworth G, Dash A, Beer Stolz D, Tannenbaum S, Griffith L and Wells A: Novel three-dimensional organotypic liver bioreactor to directly visualize early events in metastatic progression. Adv Cancer Res 97: 225-246, 2007.

30. van der Pluijm G: Epithelial plasticity, cancer stem cells and bone metastasis formation. Bone 48: 37-43, 2011.

31. Chaffer CL, Brennan JP, Slavin JL, Blick T, Thompson EW and Williams ED: Mesenchymal-to-epithelial transition facilitates bladder cancer metastasis: Role of fibroblast growth factor receptor-2. Cancer Res 66: 11271-11278, 2006.

32. Elloul S, Vaksman O, Stavnes HT, Trope CG, Davidson B and Reich R: Mesenchymal-to-epithelial transition determinants as characteristics of ovarian carcinoma effusions. Clin Exp Metastasis 27: 161-172, 2010

33. Moen I, Øyan AM, Kalland KH, Tronstad KJ, Akslen LA, Chekenya M, Sakariassen PO, Reed RK and Stuhr LE: Hyperoxic treatment induces mesenchymal-to-epithelial transition in a rat adenocarcinoma model. PLoS One 4: e6381, 2009.

34. Hill RP, Marie-Egyptienne DT and Hedley DW: Cancer stem cells, hypoxia and metastasis. Semin Radiat Oncol 19: 106-111, 2009.

35. Phinney DG: Twist, epithelial-to-mesenchymal transition, and stem cells. Stem Cells 29: 3-4, 2010.

36. Martin A and Cano A: Tumorigenesis: Twist1 links EMT to selfrenewal. Nat Cell Biol 12: 924-925, 2010.

37. Zavadil J: A spotlight on regulatory networks connecting EMT and cancer stem cells. Cell Cycle 9: 2927-2935, 2010. 